\title{
BRCA1 protein levels and $P I K 3 C A$ mutations as predictive biomarkers for response to neoadjuvant chemotherapy in locally advanced breast cancer: An exploratory analysis
}

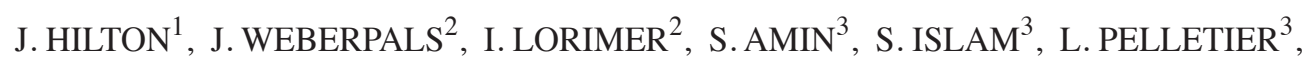 \\ M. DANESHMAND ${ }^{2}$, J. HANSON ${ }^{2}$, M.NABAVI ${ }^{3}$, D. PAROLIN ${ }^{2}$, R. MALLICK ${ }^{4}$ and S. VERMA ${ }^{1}$ \\ ${ }^{1}$ Department of Medical Oncology; ${ }^{2}$ Ottawa Hospital Research Institute; and ${ }^{3}$ Department of Pathology, Ottawa Hospital; \\ ${ }^{4}$ Department of Biostatistics, Ottawa Hospital Research Institute, University of Ottawa, Ottawa, Ontario K1N 6N5, Canada
}

Received January 16, 2012; Accepted March 19, 2012

DOI: $10.3892 /$ ol.2012.697

\begin{abstract}
BRCA1 overexpression and phosphoinositide 3-kinase (PIK3CA) pathway activation are involved in the resistance to DNA damaging agents. Thus, we hypothesized that BRCA1 protein expression and activating PIK3CA mutations are potential tumor biomarkers for the chemotherapeutic response to doxorubicin/cyclophosphamide plus docetaxel in locally advanced breast cancer. Informed consent was obtained and clinical, pathological and response data were collected. BRCA1 protein expression levels were assessed by immunohistochemistry of the archived tissue by two independent pathologists. The PIK3CA mutation status was assessed by nested PCR amplification and DNA sequencing. BRCA1 protein levels and the PIK3CA mutation status were correlated with pathological complete response and a partial response or better using the Chi-square test, Fisher's exact test and logistic regression. Of the 136 eligible participants, 59 samples could be analyzed. There was a trend of relatively low levels of BRCA1 protein achieving a pathological complete response (pCR), although this was not statistically significant [odds ratio $(\mathrm{OR})=1.74 ; \mathrm{p}=0.437$ ]. Twenty-eight percent of patients had PIK3CA mutations, but no statistically significant association with $\mathrm{pCR}(\mathrm{OR}=0.977 ; \mathrm{p}=0.971)$ was noted. Neither BRCA1 protein levels $(\mathrm{OR}=1.18$; $\mathrm{p}=0.818)$ nor PIK3CA mutations $(\mathrm{OR}=1.03$; $\mathrm{p}=0.971)$ appeared to be associated with the likelihood of achieving a partial response or better from neoadjuvant chemotherapy. PIK3CA wild-type mutation status showed a trend towards an increased likelihood of not presenting with inflammatory disease $(\mathrm{OR}=5.34$; $\mathrm{p}=0.101$ ). In this exploratory study, neither BRCA1 protein expression levels nor the presence of PIK3CA mutations were significantly associated with chemotherapy response in locally
\end{abstract}

Correspondence to: Dr John Hilton, Ottawa Hospital Cancer Center, 501 Smyth Road, Ottawa, Ontario K1H 8L6, Canada

E-mail: jfhilton@hotmail.com

Key words: neoadjuvant therapy, PIK3CA mutations, BRCA1, pathological complete response, breast cancer advanced breast cancer. However, the relatively small sample size limits the overall interpretation.

\section{Introduction}

Although overall mortality from breast cancer is on the decline, a particularly challenging situation involves patients with locally advanced breast cancer (LABC). Clinically, this subgroup is divided into two distinct categories: first, patients with large clinical stage IIA, IIB and T3N1M0 tumors who are operable but require a mastectomy; and second, those who are unlikely to have a complete surgical resection due to significant tumor burden (i.e., stage TanyN2M0). Overall, patients in the second category have a poorer prognosis due to the advanced stage and aggressive nature of their disease and the high likelihood of leaving residual disease at surgery $(1,2)$.

Due to these challenges, the treatment of locally advanced disease requires the use of neoadjuvant chemotherapy to reduce the tumor burden sufficiently to facilitate surgical resection. The National Surgical Adjuvant Breast and Bowel Project (NSABP) B18 and B27 trials demonstrated an improved relapse survival rate in patients who achieved a pathological complete response (pCR) compared to patients who did not achieve pCR, with the combination of doxorubicin and cyclophosphamide followed by docetaxel (AC-D) achieving a pCR in $26 \%$ of patients $(3,4)$. At present, there is no method to distinguish patients who is likely to respond to neoadjuvant chemotherapy; therefore, it is current practice to initiate treatment and assess the patient clinically for a response. Patients who fail to demonstrate an adequate response are administered salvage treatment, either with alternative chemotherapy agents or radiotherapy. However, this salvage approach is unlikely to benefit patients. The NSABP B27, Aberdeen and GEPARTRIO studies showed that patients who were non-responders to initial anthracycline therapy were unlikely to achieve a pCR through the addition of other cytotoxic agents (4-6). This lack of benefit is of concern as a number of neoadjuvant regimens result in significant toxicity. For example, use of AC-D in NSABP B27 yielded a $23.7 \%$ grade $4-5$ toxicity rate (7). In addition, there are concerns of potential cardiac toxicity due to the administration of anthracyclines (8). 
Response to chemotherapy is likely dependent on the molecular profile of a patient's tumor. Identification of biomarkers that predict response to neoadjuvant chemotherapy may prevent unnecessary exposure to cytotoxic agents and lead to the initiation of either experimental regimens or radiotherapy more rapidly, potentially leading to better outcomes. One possible molecular marker is breast cancer 1 (BRCA1) expression. BRCAl is a well-known tumor suppressor gene with carriers at high risk of developing breast and ovarian cancer. Preclinical evidence suggests that BRCA1 expression is a potential prognostic or predictive marker for chemotherapeutic response since functional BRCA1 is able to increase the efficacy of DNA repair (9). Studies have shown that in tumors with functional BRCA1 protein, increased BRCA1 mRNA levels are associated with reduced chemotherapy efficacy and poorer outcomes (10). Reduced intracellular BRCA1 protein levels appear to be a common finding in human breast cancer, as indicated in 30 of 108 tumor samples in a Japanese breast cancer cohort (11). Based on these findings, the expression of BRCA1 may affect response and outcomes to neoadjuvant AC-D chemotherapy.

Another candidate molecular marker is PIK3CA mutation status. PIK3CA encodes a catalytic subunit of the class I phosphoinositide-3-kinases (PI3K). PIK3CA is mutated in approximately $25 \%$ of breast cancers with activating mutations in exons 9 and 20 of PIK3CA generating constitutively active forms of the PI3-kinase (12). Hyperactivation is involved in chemoresistance through the stimulation of cell survival pathways through Akt and mTOR (13). Although results of recent studies have shown that numerous tumors with PIK3CA mutations are less aggressive, tending to present at an earlier stage of disease (14), Papaxoinis et al have reported that PIK3CA mutations were a negative prognostic factor for survival in patients presenting with high-risk breast cancer requiring chemotherapy, possibly due to chemoresistance (15). Due to its potential role in chemoresistance, activating PIK3CA mutations may affect response to neoadjuvant chemotherapy and the likelihood of achieving a pCR.

Currently, little data is available as to whether BRCA1 protein levels or PIK3CA mutations are predictive for $\mathrm{pCR}$ when AC-D was utilized in the neoadjuvant setting. Therefore, we conducted a retrospective exploratory analysis to address whether: i) lower levels of BRCA1 protein in breast cancer cells were associated with improved AC-D response; and ii) activating PIK3CA mutations were associated with AC-D resistance.

\section{Materials and methods}

Patient samples. This is a retrospective analysis which compared chemotherapy response in patients to the protein level of BRCA1 and PIK3CA mutation status. The study design was reviewed by the Ottawa Hospital Research Institute Research Ethics Board and was given approval prior to proceeding. Three criteria were established for inclusion in this study: i) A confirmed diagnosis of LABC or IBC via core biopsy prior to initiation of any therapy; ii) a clear clinical history regarding the chemotherapy regimens undertaken by the patient, and iii) overall response. Potential participants were identified using the Ottawa Hospital Cancer Centre Breast
Cancer database between 2006 and 2008. Informed patient consent for use of their tissue in this study was obtained from potential participants. Individuals who agreed to participate in the study were enrolled while those who refused participation were not contacted further by the study team.

Individuals who met the inclusion criteria and consented to participate had their clinical data derived from hospital records available at the Ottawa Hospital Cancer Centre. Clinical data of interest included patient demographics, hormone receptor status, HER-2 status, and clinical stage. Response to AC-D chemotherapy was recorded as per the RECIST criteria, a standardized, peer-reviewed reporting system designed to assess chemotherapy response (16). Tumor samples were then requisitioned from pathology, either from within the Ottawa Hospital system or externally if the biopsy was performed elsewhere.

BRCA1 protein analysis. Formalin-fixed, paraffin-embedded patient tumor samples were collected and immunohistochemical (IHC) analysis and scoring were conducted as described in a previous study (17). Formalin-fixed, paraffin-embedded blocks representing areas of tumor were retrieved from the archives of the Department of Pathology and Laboratory Medicine. Step sections were obtained from these blocks for $\mathrm{H} \& \mathrm{E}$ and IHC analysis for BRCA1 protein. IHC was performed in batches to maintain uniformity in the intensity and distribution of staining. The scoring method was adapted from the Allred and Quick scoring methods used for the scoring of estrogen and progesterone receptor immunoreactivity in breast carcinomas (18). The scores for distribution were represented as the percentage of tumor cell nuclei that stained positive from 0 to 3 ( 0 , negative; $1,<30 \% ; 2,30-70 \%$ and $3,>70 \%)$. Staining intensity was also scored from 0-3 (0, negative; 1 , mild; 2 , moderate and 3 , strong). Thus, we were able to pool cases with low intensity ( 0 or 1$)$ or distribution ( 0 or 1$)$ as negative (comprehensive scores 0 or 1 ). Cases with higher (2 or 3 ) scores in either distribution or intensity received higher comprehensive scores. Pathologists were blinded to each other's scores and to the clinical outcome data.

PIK3CA mutation analysis. Sections $(5 \mu \mathrm{M})$ from paraffinembedded specimens were subjected to either macrodissection or laser capture microdissection to ensure that all DNA samples were derived from $>80 \%$ cancer cells. DNA was isolated using QuickExtract DNA extraction kit (Epicentre Biotechnologies, Madison, WI, USA). Regions of PIK3CA exons 9 and 20 were amplified using nested PCRs (primers available from corresponding author on request). PCR products from the second round of the nested PCR were screened for the presence of mutations using high resolution melting in a Corbett Rotorgene 6000. For PCR products with melting curve deflections, the presence and specific nature of mutations was confirmed by dideoxy-sequencing. PIK3CA was considered mutant only if the well-characterized mutations E542K, E545K and H1047R were detected.

Statistical analysis. Primary statistical questions were posed for the exploratory analysis. These included whether the i) reduced levels of BRCA1 protein within the tumor specimen would correlate with an increased likelihood of achieving a 
Table I. Baseline characteristics of participants $(n=59)$.

\begin{tabular}{lr}
\hline Age (mean/median) & 51.6 years/53 years \\
Hormone receptor status & $72.8 \%$ \\
ER-positive & $61.0 \%$ \\
PR-positive & $28.8 \%$ \\
HER2 overexpression status & \\
Tumor grade & $6.8 \%$ \\
Grade 1 & $47.5 \%$ \\
Grade 2 & $45.7 \%$ \\
Grade 3 & 2 \\
Median & $20.3 \%$ \\
Participants with inflammatory disease & \\
Histological subtype & $91.5 \%$ \\
Ductal & $8.5 \%$ \\
Lobular & \\
Response to neoadjuvant AC-D & $15.3 \%$ \\
Stable disease & $55.9 \%$ \\
Partial response & $23.7 \%$ \\
Pathological complete response & $5.1 \%$ \\
Progressive disease & \\
\hline
\end{tabular}

pCR when AC-D chemotherapy was utilized; ii) a significant difference would be found in the likelihood of achieving a pCR based on the PIK3CA mutation status when AC-D chemotherapy was utilized; iii) BRCA1 protein levels and PIK3CA mutations were associated with achieving a partial response or better when AC-D chemotherapy was utilized; and iv) the protein level of BRCA1 or PIK3CA mutation status would affect the likelihood of being diagnosed with inflammatory breast cancer.

For BRCA1 protein levels, initial scoring occurred in a range from $0-9$, with 0 representing absent protein and 9 representing high levels of protein. In order to create a dichotomous variable of relatively 'high' and 'low' BRCA1 expression, a minimal p-value test was conducted. Based on this analysis, tumor samples scored as $\leq 4$ were considered to have low levels of BRCA1 protein, while samples scored $>4$ were considered to have high levels of protein. BRCA1 protein levels were then tested for correlation with pCR and a partial response or better using a Chi-square test and logistic regression analysis. For PIK3CA mutations, correlation with pCR and a partial response or better was performed using a Fisher's exact test and a logistic regression analysis. For the evaluation of whether BRCA1 protein levels and PIK3CA mutations affect the likelihood of developing inflammatory disease, exact logistic regression was used.

\section{Results}

Patient characteristics. For the years 2006-2008, the Ottawa Hospital Cancer Centre breast cancer database identified 136 eligible participants who met the inclusion criteria. A total of 65 individuals consented to participate; however, only 59 samples could be analyzed. Participant baseline characteristics

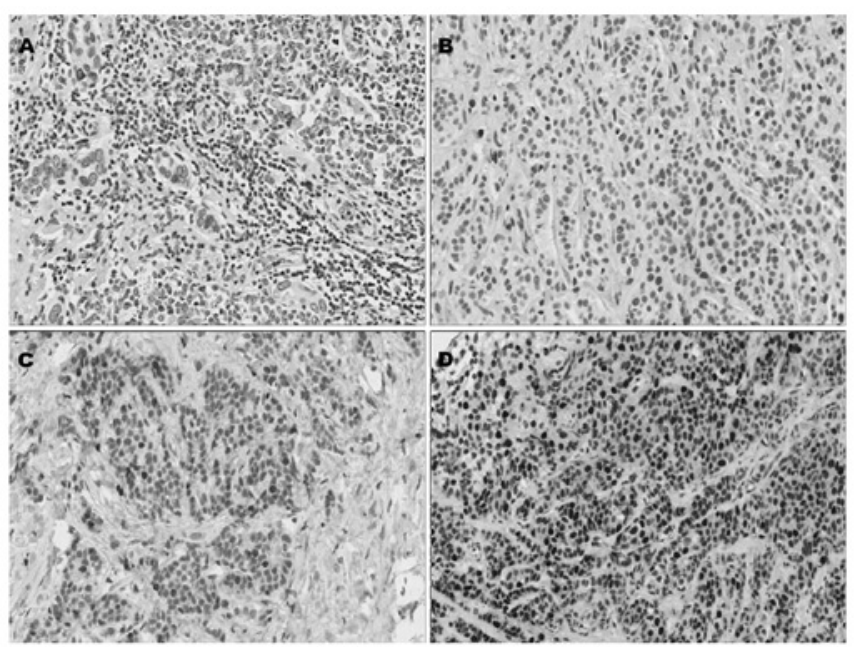

Figure 1. BRCA1 protein staining is shown. Representative immunohistochemical analysis of BRCA1 protein expression on breast cancer patient samples. BRCA1 expression was variable in the tumors analyzed with some tumors exhibiting no nuclear staining, while other tumors demonstrated significant presence of BRCA1 staining. Staining of tumors was compared to both human tissue controls and cell line controls (BRCA1-positive MCF7, BRCA1-negative UWB1.289). Staining scores: (A) 0; (B) 2; (C) 4; (D) 9.

are shown in Table I. The participants were administered AC-D chemotherapy followed by 1 year of trastuzumab initiated with docetaxel if HER2 was positive, with the exception of one participant who received 6 cycles of carboplatin/taxotere due to a pre-existing cardiac issue. Partial response or better to therapy was observed in $68.3 \%$ of participants, with $23.7 \%$ achieving a pCR.

BRCA1 protein staining from tumor samples. In tumor cells, immunostaining for BRCA1 was mostly nuclear, with occasional cytoplasmic hue in certain samples. Normal appearing breast epithelial cells adjacent to the tumor did not show any immunostaining, although occasional nuclei in myoepithelial cells showed strong immunoreactivity (Fig. 1). Based on the dichotomous variable of relatively 'high' and 'low' levels of BRCA1 expression established by the minimal p-value test ( $\leq$ 4 vs. > 4), 33 samples were scored as having 'low' levels of BRCA1 expression, while 21 samples were scored as having 'high' levels of BRCA1 expression.

Correlation of PIK3CA mutations and BRCA1 protein expression to chemotherapy response. With regards to PIK3CA mutations, $27 \%$ of patients had a mutation in either exon 9 or 20 , with exon 20 mutations being the most common (18\%). This is similar to the mutation frequencies that have been reported for breast cancer as a whole $(19,20)$. The presence of a mutation did not appear to affect the likelihood of achieving a pCR to neoadjuvant chemotherapy compared to tumor samples possessing a wild-type $P I K 3 C A$ mutation $(\mathrm{OR}=0.977$; $\mathrm{p}=1.00)$. A similar observation was found when $P I K 3 C A$ mutations were evaluated against achieving a partial response or better $(\mathrm{OR}=1.03$; $\mathrm{p}=0.971)$ (Table II).

A relatively low level of BRCA1 protein expression was more likely associated with achieving a pCR compared to a high level of BRCA1 expression, although this result was 
Table II. Correlations of BRCA1 protein level and PIK3CA mutations to pCR.

Statistical comparison

Result

PI3KCA mutation status and $\mathrm{pCR}$

No association

$\mathrm{OR}=0.977$ if

wild type $(\mathrm{p}=1.00)$

PI3KCA mutation status and partial response or better

No association $\mathrm{OR}=1.03$ if wild type $(\mathrm{p}=0.971)$

BRCA1 protein level and $\mathrm{pCR}$

No association $\mathrm{OR}=1.74$ if low $(\mathrm{p}=0.44)$

BRCA1 protein level and partial response or better

No association $\mathrm{OR}=1.18$ if low $(\mathrm{p}=0.82)$

not statistically significant (OR 1.74; $\mathrm{p}=0.44$ ). In addition, low levels of BRCA1 protein expression do not appear to be associated with the likelihood of achieving a partial response or better to neoadjuvant chemotherapy $(\mathrm{OR}=1.18 ; \mathrm{p}=0.82)$ (Table II).

Participants with PIK3CA wild-type proteins were more likely to be diagnosed with inflammatory disease compared to those with mutations in $P I K 3 C A$, but this finding did not reach statistical significance $(O R=5.34$ for wild type; $p=0.109)$. Participants with low levels of BRCA1 protein were at an increased risk of being diagnosed with inflammatory disease, but this finding did not reach statistical significance $(\mathrm{OR}=2.86$; $\mathrm{p}=0.407$ ).

\section{Discussion}

Inoperable locally advanced disease is a challenging subtype of potentially curable breast cancer. As cure is dependent on achieving a complete surgical excision of the primary tumor, patients with inoperable disease require neoadjuvant chemotherapy in the hope of reducing the disease burden significantly enough to allow for surgery to occur. In addition, the NSABP B18 and B27 trials have demonstrated an improved relapse survival rate in patients who achieve a pCR when neoadjuvant chemotherapy is utilized $(3,4)$. By contrast, other women present with operable disease but opt to undergo neoadjuvant chemotherapy to allow breast conserving surgery to be performed. In all of these cases, predictive biomarkers for response to neoadjuvant chemotherapy may allow treating physicians to predict who is likely to benefit from this approach and possibly select other treatment modalities or clinical trial protocols in the hope of benefitting their patients.

In this exploratory study, we evaluated PIK3CA mutations and intracellular BRCA1 protein levels to see whether they are associated with response to neoadjuvant chemotherapy. Preclinical evidence suggests that the two factors may affect response to chemotherapy. PIK3CA mutations in breast cancer lead to increased PI3K pathway signalling, which increased the activity of the Akt and mTOR proteins, leading to increased cell survival and resistance to cancer therapy (14). Increased BRCA1 protein levels are able to increase the efficacy of DNA repair in the presence of chemotherapy $(9,21)$. Thus, we hypothesized that these two factors may be associated with chemotherapy resistance and be worthy of exploration in the neoadjuvant setting.

In our study, no statistical association was identified between PIK3CA mutations or BRCA1 protein levels and response to neoadjuvant chemotherapy in patients with LABC. This was an exploratory study with a limited number of available samples for analysis. However, results of our study suggest trends for each of these factors to provide a foundation for a larger, prospective study. In terms of PIK3CA mutations, such a trend is clearly not present for either the achievement of $\mathrm{pCR}(\mathrm{OR}=0.977, \mathrm{p}=1.00)$ or response to chemotherapy $(\mathrm{OR}=1.03, \mathrm{p}=0.971)$. This result is noteworthy, given the strong preclinical rationale for this pathway in terms of chemotherapy resistance. Notably, PIK3CA mutations appeared to associate with the type of disease at presentation. Patients with activating mutations were much less likely to present with inflammatory disease, an aggressive subtype of LABC with a poor prognosis. Although to the best of our knowledge this is the first study to examine PIK3CA mutations specifically in LABC, the data obtained are consistent with previous studies in which PIK3CA mutations are associated with hormone receptor positivity and less aggressive disease (14).

Low BRCA1 protein levels appear to be more promising in predicting for $\mathrm{pCR}$, but the current results are not definitive $(\mathrm{OR}=1.71, \mathrm{p}=0.44)$. Given that $\mathrm{BRCA} 1$ is a predictive and prognostic factor for improved chemotherapy response and outcome in sporadic ovarian cancer (20), more studies with a larger sample size are required.

There are certain limitations to our study. First, our sample size was small, reducing the overall power. It is possible that clearer associations could have been detected with a larger data set. Second, although we adopted a generalized approach, it is possible that had we focused on only particular pathological features, such as ER-positive or HER2-positive, a more distinct association might have been detected. However, the population group included in this study was insufficient for these specific subgroup analyses.

In conclusion, results of our exploratory study did not show that PIK3CA mutations or BRCA1 protein levels are associated with response to neoadjuvant chemotherapy in patients with LABC, although the small sample size limits the conclusions that may be drawn. Thus, the role of BRCA1 protein levels in chemotherapy response should be investigated, given that there was a trend towards greater response in tumors expressing low levels of the protein and evidence of correlation with chemotherapeutic response in ovarian cancer. Such studies should be prospective and a larger number of participants should be included.

\section{Acknowledgements}

This study was possible due to generous funding provided by the Ottawa Regional Cancer Foundation. 


\section{References}

1. Gralow JR, Burstein HJ, Wood W, et al: Preoperative therapy in invasive breast cancer: pathologic assessment and systemic therapy issues in operable disease. J Clin Oncol 26: 814-819, 2008.

2. Kleer CG, van Golen KL and Merajver SD: Molecular biology of breast cancer metastasis. Inflammatory breast cancer: clinical syndrome and molecular determinants. Breast Cancer Res 2: 423-429, 2000

3. Fisher B, Bryant J, Wolmark N, et al: The effect of preoperative chemotherapy on the outcome of women with operable breast cancer. J Clin Oncol 16: 2672-2685, 1998

4. Bear HD, Anderson S, Smith RE, et al: Sequential preoperative or postoperative docetaxel added to preoperative doxorubicin plus cyclophosphamide for operable breast cancer: national surgical adjuvant breast and bowel project protocol B-27. J Clin Oncol 24: 2019-2027, 2006.

5. Smith IC, Heys SD, Hutcheon AW, et al: Neoadjuvant chemotherapy in breast cancer: significantly enhanced response with docetaxel. J Clin Oncol 20: 1456-1466, 2002.

6. Von Minckwitz G, Blohmer JU, Raab G, et al: In vivo chemosensitivity-adapted preoperative chemotherapy in patients with early-stage breast cancer: the GEPARTRIO pilot study. Ann Oncol 16: 56-63, 2005.

7. Bear HD, Anderson S, Brown A, et al: The effect on tumor response of adding sequential preoperative docetaxel to preoperative doxorubicin and cyclophosphamide: preliminary results from national surgical adjuvant breast and bowel project protocol B-27. J Clin Oncol 21: 4165-4174, 2003.

8. Smith LA, Cornelius VR, Plummer CJ, et al: Cardiotoxicity of anthracycline agents for the treatment of cancer: systematic review and meta-analysis of randomised controlled trials. BMC Cancer 10: 337-351, 2010.

9. Weberpals JI, Clark-Knowles KV and Vanderhyden BC: Sporadic epithelial ovarian cancer: clinical relevance of BRCA1 inhibition in the DNA damage and repair pathway. J Clin Oncol 26: 3259-3267, 2008.
10. Gennari A, Sormani M, Varesco L, et al: Prognostic significance of BRCA1, PARP1 and PARP2 in sporadic breast cancer. J Clin Oncol 27s: e22114, 2009.

11. Yoshikawa K, Honda K, Inamoto T, et al: Reduction of BRCA1 protein expression in Japanese sporadic breast carcinomas and its frequent loss in BRCA1-associated cases. Clin Cancer Res 5: 1249-1261, 1999.

12. Zhao L and Vogt PK: Class I PI3K in oncogenic cellular transformation. Oncogene 27: 5486-5496, 2008.

13. Yuan TL and Cantley LC: PI3K pathway alterations in cancer: variations on a theme. Oncogene 27: 5497-5510, 2008.

14. Kalinsky K, Jacks LM, Heguy A, et al: PIK3CA mutation associates with improved outcome in breast cancer. Clin Cancer Res 15: 5049-5059, 2009.

15. Papaxoinis G, Pectasides D, Wirtz RM, et al: Prognostic significance of PI3K mRNA expression in patients with operable, high risk breast cancer. J Clin Oncol 27s: 577, 2009.

16. Eisenhauer EA, Therasse P, Bogaerts J, et al: New response evaluation criteria in solid tumors: revised RECIST guideline (version 1.1). Eur J Cancer. 45: 228-247, 2009.

17. Weberpals JI, Tu D, Squire JA, et al: Breast cancer 1 (BRCA1) protein expression as a prognostic marker in sporadic epithelial ovarian carcinoma: an NCIC CTG OV.16 correlative study. Ann Oncol 22: 2403-10, 2011.

18. Allred DC, Harvey JM, Berardo M and Clark GM: Prognostic and predictive factors in breast cancer by immunohistochemical analysis. Mod Pathol 11: 155-168, 1998.

19. Bader AG, Kang S, Zhao L and Vogt PK: Oncogenic PI3K deregulates transcription and translation. Nat Rev Cancer 5: 921-929, 2005.

20. Karakas B, Bachman KE and Park BH: Mutation of the PIK3CA oncogene in human cancers. Br J Cancer 94: 455-459, 2006.

21. Carser JE, Quinn JE, Michie CO, et al: BRCA1 is both a prognostic and predictive biomarker of response to chemotherapy in sporadic epithelial ovarian cancer. Gynecol Oncol 123: 492-498, 2011 